Article

\title{
Determination and Dissection of DNA-Binding Specificity for the Thermus thermophilus HB8 Transcriptional Regulator TTHB099
}

\author{
Kristi Moncja $@$ and Michael W. Van Dyke *(1) \\ Department of Chemistry and Biochemistry, Kennesaw State University, Kennesaw, GA 30144, USA; \\ kmoncja@students.kennesaw.edu \\ * Correspondence: mvandyk2@kennesaw.edu; Tel.: +1-470-578-2793
}

Received: 14 October 2020; Accepted: 24 October 2020; Published: 26 October 2020

\begin{abstract}
Transcription factors (TFs) have been extensively researched in certain well-studied organisms, but far less so in others. Following the whole-genome sequencing of a new organism, TFs are typically identified through their homology with related proteins in other organisms. However, recent findings demonstrate that structurally similar TFs from distantly related bacteria are not usually evolutionary orthologs. Here we explore TTHB099, a cAMP receptor protein (CRP)-family TF from the extremophile Thermus thermophilus HB8. Using the in vitro iterative selection method Restriction Endonuclease Protection, Selection and Amplification (REPSA), we identified the preferred DNA-binding motif for TTHB099, 5'-TGT(A/g)NBSYRSVN(T/c)ACA-3', and mapped potential binding sites and regulated genes within the T. thermophilus HB8 genome. Comparisons with expression profile data in TTHB099-deficient and wild type strains suggested that, unlike E. coli CRP (CRP $\left.\mathrm{Ec}_{\mathrm{Ec}}\right)$, TTHB099 does not have a simple regulatory mechanism. However, we hypothesize that TTHB099 can be a dual-regulator similar to $\mathrm{CRP}_{\mathrm{Ec}}$.
\end{abstract}

Keywords: bioinformatics; biolayer interferometry (BLI); electrophoretic mobility shift assay (EMSA); extremophile; protein-DNA binding; type IIS restriction endonuclease

\section{Introduction}

Transcription factors (TFs) are DNA-binding proteins that allow for modulation of transcription initiation in response to intracellular and extracellular changes. Over decades of research, there have been many advances in exploring the TFs regulatory mechanisms cells use to control their gene expression. However, technological innovations such as massively parallel sequencing and data sciences have expanded our interest in new model organisms and their adaptations. TFs are trans factors that bind to cis-regulatory elements, promoter or enhancer sequences known as TF binding sites (TFBSs). It has been reported that most of the bacterial TFBSs are found in the proximal region (about -100 to $+20 \mathrm{bp}$ from the transcription start site [TSS]) and distal regions (up to -200 from TSS) [1-3]. Functionally, TFs are categorized into activators and suppressors, with a few of them being dual-regulators [4]. Regarding the number of genes regulated, TFs are classified into local or global regulators [5]. Such characteristics make up the mechanism of transcription regulation and help identify novel TFs.

Proteomic studies allow the grouping of TFs into families based on structural comparison studies. However, new findings have shown that structurally similar TFs from distantly related bacteria are not usually evolutionary orthologs [6]. A more comprehensive characterization of the TF regulatory network is achieved by identifying the TFBSs, the genes regulated, and the method of regulation. Advances in computational biology and data processing have given rise to inclusive databases that can 
predict structure and function for TFs in new model organisms [7]. However, most of these databases are built from experimental studies.

To gain insights into transcriptional regulatory networks in extremophile organisms, our laboratory has employed a novel biochemistry-based method, Restriction Endonuclease, Selection, Protection, and Amplification (REPSA), to characterize several TFs in the extreme thermophilic model organism Thermus thermophilus HB8. To date, we have studied four tetracycline repressor protein (TetR) family transcriptional suppressors and have successfully identified their TFBSs [8-11]. Commonly, suppressors bind DNA in the absence of small-molecule modulators/cofactors and with high-affinity. Contrary, numerous transcriptional activators employ small-molecule modulators in order to bind DNA, thus complicating their analysis in vitro.

In this study, we explore the utility of REPSA to identify and characterize a potential thermophilic transcriptional activator, TTHB099. Protein sequence homology analysis indicates that TTHB099 is one of the four cAMP receptor protein (CRP) family members (TTHA1437, TTHA1359, TTHB099, and TTHA1567) in T. thermophilus HB8 and should bind palindromic DNA sequences as a homodimer [12]. However, despite having a cAMP binding domain, it does not require this cofactor to bind DNA. Here, we identified the preferred DNA-binding sequence for TTHB099 as the 16-mer motif: $5^{\prime}-\mathrm{TGT}(\mathrm{A} / \mathrm{g}) \mathrm{n}(\mathrm{t} / \mathrm{c}) \mathrm{c}(\mathrm{t} / \mathrm{c})(\mathrm{a} / \mathrm{g}) \mathrm{g}(\mathrm{a} / \mathrm{g}) \mathrm{n}(\mathrm{T} / \mathrm{c}) \mathrm{ACA}-3^{\prime}$. Furthermore, we used binding kinetics studies and mRNA expression data to validate potential biological roles of TTHB099.

\section{Results}

\subsection{Preferred TTHB099-Binding Sequences Selected Via REPSA}

REPSA was used to select for TTHB099-binding sites present in a large pool ( 60 billion molecules) of synthesized double-stranded DNA. Our selection library, ST2R24, has been successfully used in four previous studies [8-11]. IRDye ${ }^{\circledR} 700$ (IRD7)-labeled library DNA was incubated with purified TTHB099 protein to permit specific binding, then challenged by a type IIS restriction endonuclease (IISRE). Sequence-specific binding of TTHB099 to a subset of the library protected those oligonucleotides from endonuclease activity, thereby permitting their amplification by PCR. Seven rounds of binding, IISRE cleavage, and PCR resulted in the enrichment of DNA resistant to IISRE cleavage when TTHB099 was present (Figure 1, Round 7). Note that in Round 4, substantial uncut DNA appeared on the IISRE control lane $(-/ F)$ as well as the test lane $(+/ F)$. This nonspecific cleavage inhibition has been observed before and has been ascribed to the selection of FokI cleavage-resistant sequences $[8,13]$. Thus, subsequent rounds of REPSA were performed with an alternative, albeit less efficient IISRE (BpmI).

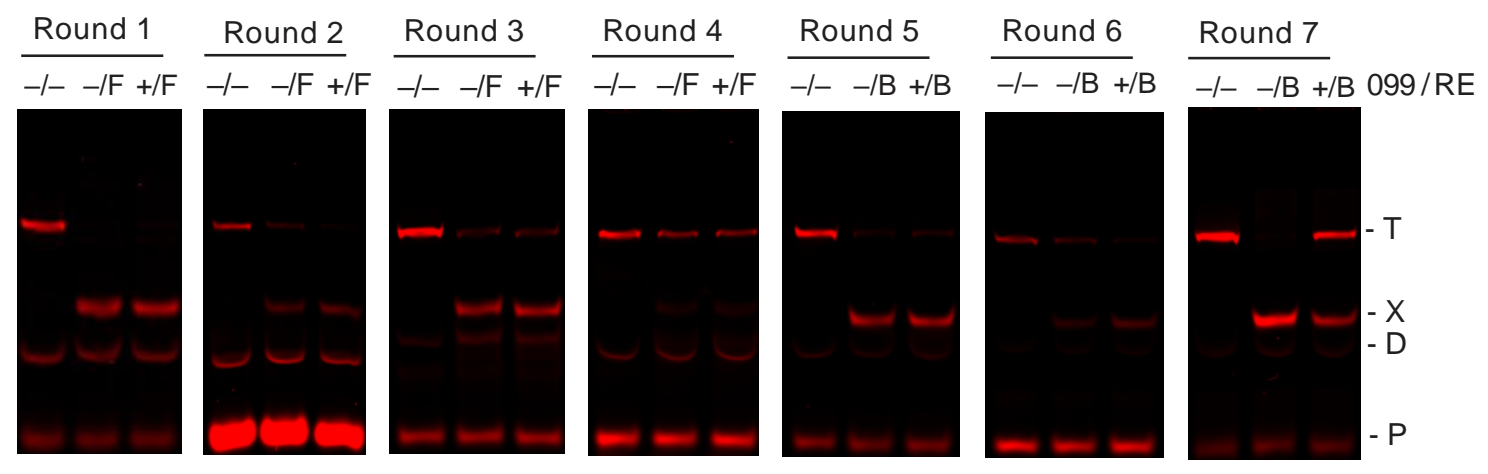

Figure 1. Selection of TTHB099-binding DNA sequences. Shown are IR fluorescence images of restriction endonuclease cleavage-protection assays made during Rounds 1-7 of REPSA selection with $50.6 \mathrm{nM}$ TTHB099 protein. The presence (+) or absence (-) of TTHB099 and IISRE FokI (F) or BpmI (B) are indicated above each lane. The electrophoretic mobility of the intact (T) and cleaved (X) ST2R24 selection template, primer dimer species (D), as well as the IRD7_ST2R primer (P) are indicated at the right of the figure. 
Before proceeding, it is prudent to validate our selection of TTHB099-binding sequences. To do so, REPSA-selected DNA was subjected to a restriction endonuclease protection assay (REPA), which is very much like the binding and IISRE cleavage steps of REPSA [14]. The inclusion of a different fluorophore-labeled control DNA in these reactions permitted the discrimination of TTHB099-specific and nonspecific IISRE cleavage inhibition. Thus, Round 7 DNA exhibited the expected pattern of cleavage protection expected for a majority population of TTHB099-binding DNA (Figure 2A). However, Round 4 DNA exhibited TTHB099-independent cleavage protection of the selected DNA, consistent with a majority being refractory to cleavage by the IISRE FokI. Additional validation was achieved using an electrophoretic mobility shift assay (EMSA) to directly visualize TTHB099-DNA complexes. In this independent assay, different concentrations of TTHB099 protein were incubated with Round 1 and Round 7 DNA prior to electrophoresis (Figure 2B). The slower mobility of the DNA-protein complex present in Round 7 but not in Round 1 DNA indicated that a substantial portion of the selected sequences contained stable TTHB099 binding sites. The results from REPSA and EMSA encouraged further studies on determining TTHB099-DNA binding sequences.

A

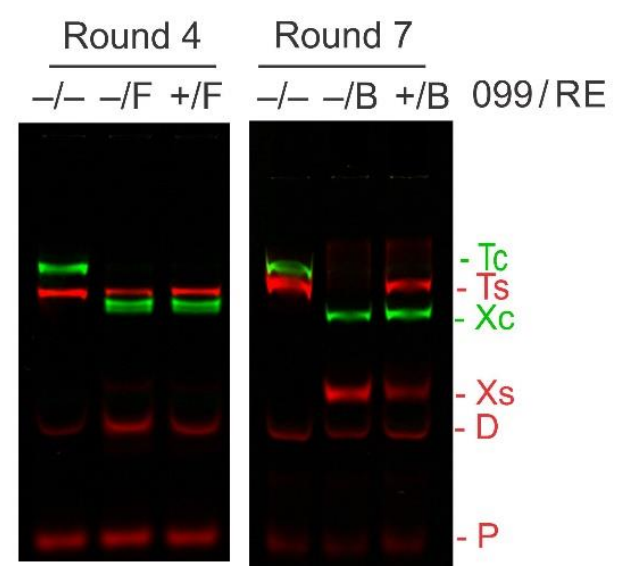

B

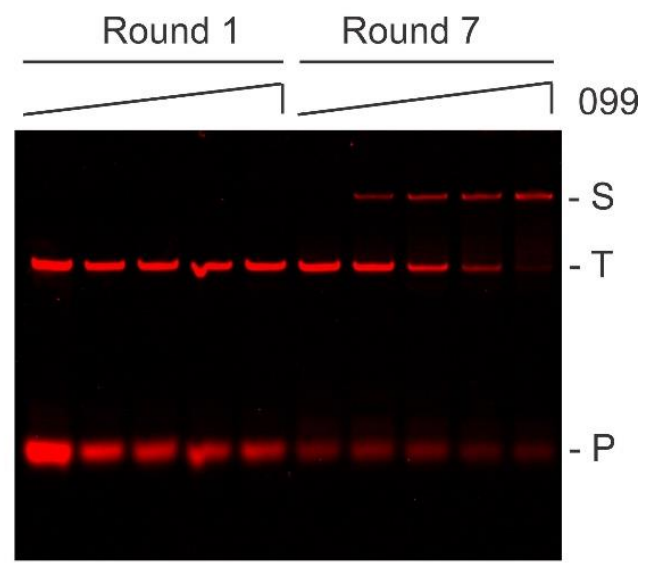

Figure 2. Validation of TTHB099-binding DNA sequences. (A) Shown are IR fluorescence images of restriction endonuclease protection assays made with DNA from Round 4 and 7 of REPSA selection. The presence (+) or absence (-) of TTHB099 and IISRE FokI (F) or BpmI (B) are indicated above each lane. The electrophoretic mobility of the intact $(\mathrm{T})$ and cleaved $(\mathrm{X})$ IRD8-labeled REPSAis control DNA (green, $T_{c}$ and $X_{c}$ ), IRD7-labeled ST2R24 selection template (red, $T_{s}$ and $X_{s}$ ), primer-dimers (D), as well as the IRD7_ST2R primer (P) are indicated at the right of the figure and color-coded to match the fluorescently labeled DNA present. (B) Shown are IR fluorescence images of electrophoretic mobility shift assays made with DNA mixtures obtained from Round 1 (left lanes) and Round 7 (right lanes) of REPSA selection incubated with increasing concentrations of TTHB099 protein (from left to right: 0, 5.06, 50.6, 506, and $5060 \mathrm{nM}$ TTHB099). The electrophoretic mobility of a single protein-DNA complex (S) as well as the uncomplexed ST2R24 selection template (T) and IRD7_ST2R primer (P) are indicated at the right of the figure.

\subsection{Identification and Characterization of TTHB099-Binding Motif}

To massively parallel sequence the REPSA-selected DNA, Round 7 DNA was amplified with fusion PCR primers, purified, and emulsion PCR amplified onto individual sequencing particles (ISPs). The enriched ISPs were subjected to next-generation semiconductor sequencing using an Ion Personal Genome Machine (PGM) system. The multiplexed sequencing run yielded 6,921,164 total bases, $6,169,384 \geq$ Q20, resulting in 120,585 reads of 57-bp mean length for the TTHB099 Round 7 DNA. A randomly selected set of 1000 reads was input into web version 5.0.5 of Multiple Em for Motif Elicitation (MEME) analyzed using default parameters with and without a palindromic filter [15]. The output position weight matrices displayed the best 23-mer motif without filters with an E-value 
of $2.4 \times 10^{-2234}$ (Figure 3A), and the best 16-mer palindromic motif with an E-value of $2.4 \times 10^{-2871}$ (Figure $3 \mathrm{~B}$ ). These statistically significant results indicate that the identified motifs are likely consensus sequences for the TTHB099 transcription factor.

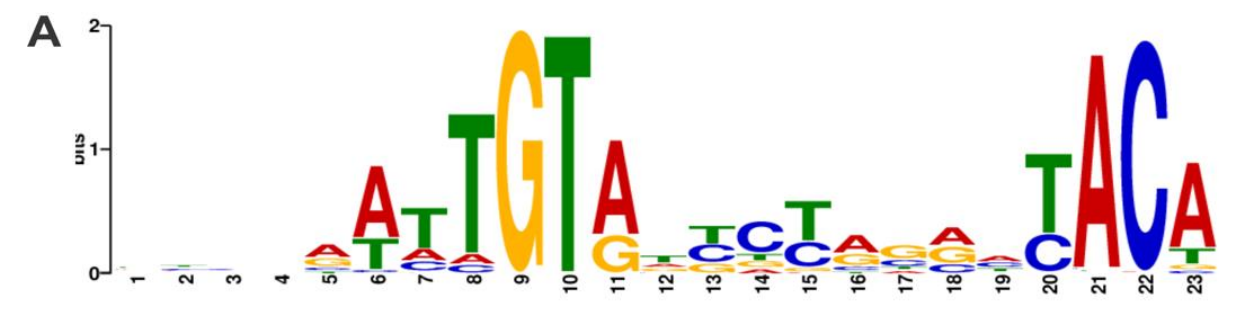

B

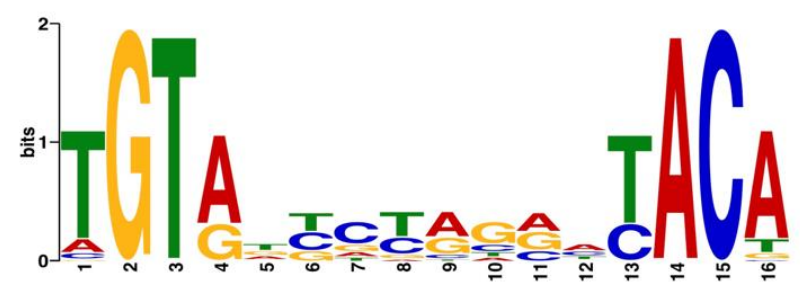

Figure 3. TTHB099-binding motifs. Sequence logos were determined using MEME software with an input of 1000 Round 7 DNA sequences. (A) MEME performed with no filters. (B) MEME performed using a palindromic filter.

Noting that the nonpalindromic sequence logo is an extended version of the palindromic one, with seven vaguely significant nucleotides upstream, it was postulated that the palindromic logo is a better representation of the TTHB099 consensus DNA-binding sequence. To test this hypothesis, the 16-mer sequence 5'-TGTATTCTAGAATACA-3' was incorporated into an ST2 background, yielding the probe ST2_099. A fixed concentration of IRD7-labeled ST2_099 was incubated with increasing purified TTHB099 protein concentrations to permit specific binding and the resulting products analyzed by EMSA (Figure 4). We found that the TTHB099-ST2_099 complex exhibited similar electrophoretic mobility as observed with the TTHB099-Round 7 DNA complex (Figure 2B), suggesting that most Round 7 DNA contained the palindromic sequence. Quantitative densitometry analysis of the fourth lane gives an approximate dissociation constant $\left(\mathrm{K}_{\mathrm{D}}\right)$ of $4.5 \mathrm{nM}$ (Supplementary Figure S1, Supplementary Table S1).

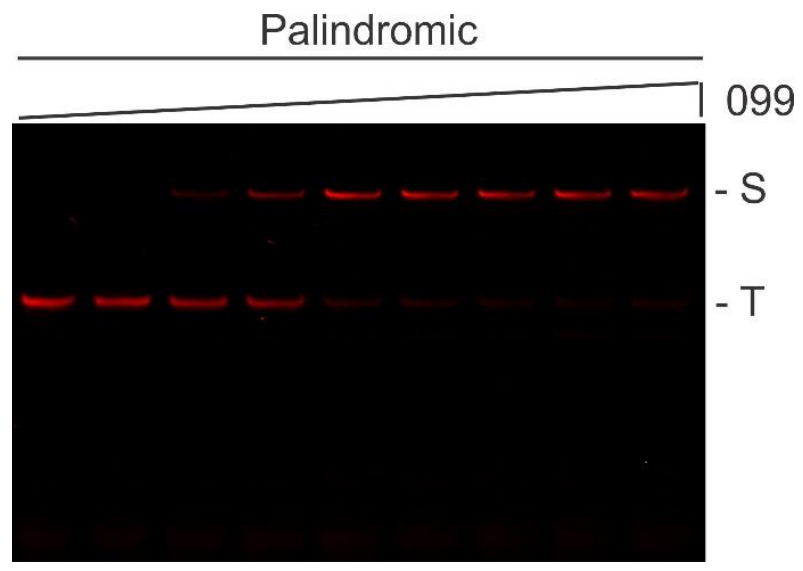

Figure 4. EMSA analysis of TTHB099 binding to its palindromic consensus sequence. Shown is an IR fluorescence image of IRD7-labeled ST2_099 incubated with 0, 0.66, 1.32, 2.64, 5.27, 10.5, 21.1, or 42.2 nM TTHB099 protein. (S) Protein-DNA complex, and (T) uncomplexed DNA. 
Biolayer interferometry (BLI) was used to characterize TTHB099-consensus DNA interactions. This innovative approach measures in vitro real-time interactions between macromolecules, including proteins and nucleic acids [16]. Our BLI analysis involved biotinylated consensus sequence, ST2_099, affixed onto streptavidin sensors interacting with increasing TTHB099 protein concentrations in solution. This provided a qualitative observation of protein-DNA association and dissociation kinetics (Figure 5A). The most substantial interactions were observed for the highest concentrations of TTHB099 (450 nM (red) and $150 \mathrm{nM}$ (green)). An arbitrary DNA sequence, ST2_REPSAis, was tested as a control DNA (Figure 5B). It demonstrated binding interactions that were below our experimental detection levels, consistent with a low TTHB099-REPSAis affinity. Another outcome of this study was the quantitative evaluation of the TTHB099-consensus binding affinity. Least squares regression analysis of the association and dissociation rates were calculated with GraphPad Prism 8. From those rates, a dissociation constant was produced. TTHB099 interacting with its consensus sequence had a $K_{D}$ of $2.214 \mathrm{nM}$ with an $\mathrm{R}^{2}$ value of 0.9883 .

A

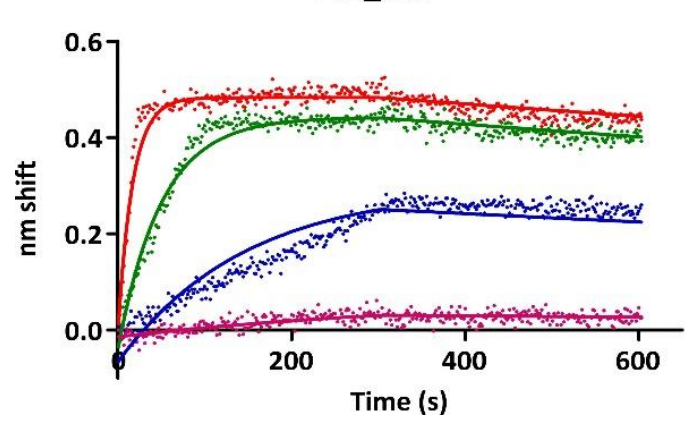

B

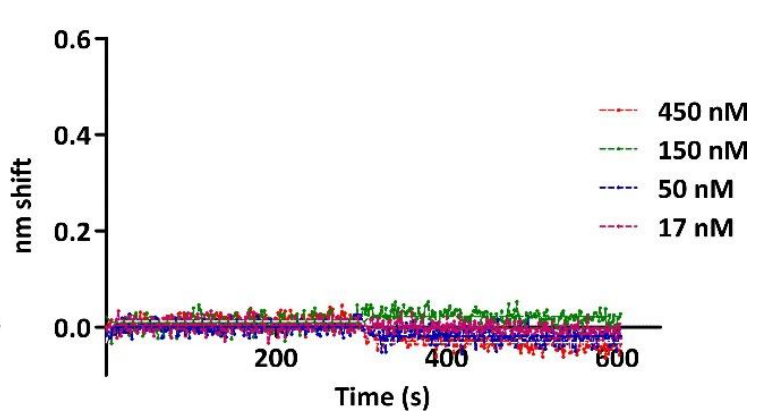

Figure 5. Biolayer interferometry analysis of TTHB099 binding to DNA. Shown are raw traces (dots) and best-fit lines of TTHB099 binding to (A) ST2_099 consensus DNA and (B) ST2_REPSAis control DNA TTHB099. Concentrations investigated include $450 \mathrm{nM}$ (red), $150 \mathrm{nM}$ (green), $50 \mathrm{nM}$ (blue), and $17 \mathrm{nM}$ (magenta).

Further characterization of TTHB099-DNA binding was made using selected point mutations of its consensus sequence and BLI. Binding kinetics data, including association rate $\left(\mathrm{k}_{\mathrm{on}}\right)$, dissociation rate $\left(\mathrm{k}_{\mathrm{off}}\right)$, and the dissociation constant, were derived for each of the mutated sequences and displayed in Table 1. As observed with the $\mathrm{m} 2$ mutant, a single change in a highly conserved nucleotide of the consensus sequence affects the binding affinity by 15 -fold. Even point mutations of less conserved positions (e.g., m5) decreased affinity by 2 -fold. These data suggest that the TTHB099 binding to DNA is highly sequence-specific. Additionally, the nanomolar dissociation constant we observed indicates that our consensus sequence is a good representation of the native TTHB099's preferred sequences in T. thermophilus HB8. Notably, TTHB099-DNA binding is not affected by the absence or presence of the second messenger $3^{\prime}, 5^{\prime} \mathrm{CAMP}$, unlike its archetype protein $\mathrm{CRP}_{\mathrm{Ec}}[17]$. 
Table 1. TTHB099-DNA binding parameters for consensus and mutant sequences.

\begin{tabular}{cccccc}
\hline Name & Sequence & $\left.\boldsymbol{k}_{\mathbf{o n}} \mathbf{( M}^{\mathbf{- 1}} \mathbf{s}^{\mathbf{- 1}}\right)$ & $\boldsymbol{k}_{\mathbf{o f f}}\left(\mathbf{s}^{\mathbf{- 1}}\right)$ & $\mathbf{K}_{\mathbf{D}} \mathbf{( M )}$ & $\mathbf{R}^{\mathbf{2}}$ \\
\hline wt & TGTATTCTAGAATACA & 131,308 & $2.907 \times 10^{-4}$ & $2.214 \times 10^{-9}$ & 0.9883 \\
m1 & gGTATTCTAGAATACA & 120,059 & $7.558 \times 10^{-4}$ & $6.295 \times 10^{-9}$ & 0.9895 \\
m2 & TtTATTCTAGAATACA & 112,773 & $3.785 \times 10^{-3}$ & $3.356 \times 10^{-8}$ & 0.9778 \\
m3 & TGaATTCTAGAATACA & 88,146 & $1.221 \times 10^{-3}$ & $1.385 \times 10^{-8}$ & 0.9824 \\
m4 & TGTCTTCTAGAATACA & 142,953 & $1.366 \times 10^{-3}$ & $9.557 \times 10^{-9}$ & 0.9817 \\
m5 & TGTAcTCTAGAATACA & 110,766 & $5.379 \times 10^{-4}$ & $4.856 \times 10^{-9}$ & 0.9879 \\
m6 & TGTATaCTAGAATACA & 125,945 & $7.064 \times 10^{-4}$ & $5.608 \times 10^{-9}$ & 0.9794 \\
m7 & TGTATTtTAGAATACA & 119,827 & $6.978 \times 10^{-4}$ & $5.823 \times 10^{-9}$ & 0.9805 \\
m8 & TGTATTCaAGAATACA & 115,299 & $7.848 \times 10^{-4}$ & $6.807 \times 10^{-9}$ & 0.9840 \\
wt + cAMP & TGTATTCTAGAATACA & 214,759 & $4.780 \times 10^{-4}$ & $2.226 \times 10^{-9}$ & 0.9231 \\
\hline
\end{tabular}

(Sequence) Lowercase nucleotides indicate a mutation from the TTHB099 consensus sequence (wt). (wt + cAMP) Binding reactions performed with the consensus sequence in the presence of $100 \mathrm{nM} 3^{\prime}, 5^{\prime} \mathrm{cAMP}$.

\subsection{T. thermophilus HB8 Genome-Wide Mapping of the TTHB099-Binding Motif}

The Find Individual Motif Occurrences (FIMO) program was used to scan the T. thermophilus HB8 genome (GenBank uid13202 210) for the 16-mer palindromic sequence identified through MEME software [18]. FIMO revealed 78 motif occurrences with a $p$-value of less than 0.0001 . The top 25 results with $p$-values $\leq 3.95 \times 10^{-5}$ are shown in Table 2. The locations of these 25 sequences relative to the transcription start site of their proximally downstream genes were determined using the Kyoto Encyclopedia of Genes and Genomes (KEGG) and verified in the National Center for Biotechnology Information (NCBI) database $[19,20]$. Furthermore, operon predictions for each location were made using the Database of PrOkaryotic OpeRons $\left(\mathrm{DOOR}^{2}\right)$ and BioCyc [21,22]. Sixteen of these sites were situated within the -200 to +20 nucleotide region most common for transcription activator binding. Furthermore, their proximally downstream genes were the first of their operons or single transcriptional units, making these sites stronger candidates for TF regulation. The other nine sites were omitted from further analysis because they were located further downstream, inside open reading frames, or, as in the case of TTHC003, too far upstream (-666 nucleotides).

To better ascertain a potential role for TTHB099 to regulate transcription, all the 16 sequences selected from FIMO were analyzed for potential core promoter elements. Sequences $\pm 200 \mathrm{bp}$ upstream and downstream of the FIMO identified TTHB099-binding sites were evaluated in SoftBerry BPROM (Figure 6) [23]. Many sequences (9/16) contained a TTHB099-consensus sequence that overlapped with at least one promoter element $(-35$ box,-10 box,+1 start site). Those included TTHA0081/80, TTHA0507, TTHA0133, TTHA1833, TTHA1912, TTHA0202, TTHA0374, and TTHA1627. Three of the TTHB099-binding sequences, TTHA0506, TTHB089, and TTHA0201, were located upstream of the nearby -35 box. Conversely, TTHB088 and TTHA1626 had their putative TTHB099-binding sequences located downstream of the postulated promoter elements. There were no identified promoter elements near TTHA0132 and TTHA1911. It is not clear why BPROM was unable to identify any core promoter elements for these genes, but limitations could arise from a potential difference between core promoter elements in E. coli, the model organism used by BPROM, and those of T. thermophilus HB8. 
Table 2. TTHB099-consensus sequences mapped in the genome of T. thermophilus HB8.

\begin{tabular}{|c|c|c|c|c|c|c|c|}
\hline Start & End & $p$-Value & $Q$-Value & Sequence & Loc & Gene & Op \\
\hline 81,408 & 81,423 & $4.03 \times 10^{-6}$ & 1 & AGTAAACTAAAACACA & +1 & TTHA0081 & $1 / 3$ \\
\hline 81,408 & 81,423 & $4.03 \times 10^{-6}$ & 1 & TGTGTTTTAGTTTACT & -48 & ТTHА0080 & S \\
\hline 32,704 & 32,719 & $5.82 \times 10^{-6}$ & 1 & TGTGTACGAAATTACA & +434 & TTHА0030 & $1 / 2$ \\
\hline 472,203 & 472,218 & $7.74 \times 10^{-6}$ & 1 & TGTATCTTGAAAAACA & -26 & TTHA0507 & S \\
\hline 472,203 & 472,218 & $7.74 \times 10^{-6}$ & 1 & TGTTTTTCAAGATACA & -56 & TTHA0506 & S \\
\hline 130,005 & 130,020 & $1.01 \times 10^{-5}$ & 1 & TTТАТТСТСССТТАСА & -10 & TTHA0133 & $1 / 2$ \\
\hline 130,005 & 130,020 & $1.01 \times 10^{-5}$ & 1 & TGTAAGGGAGAATAAA & -3 & TTHA0132 & $S$ \\
\hline 1506 & 1521 & $1.23 \times 10^{-5}$ & 1 & AGTGAGATAACTCACA & -666 & ТТНС003 & $1 / 3$ \\
\hline 1506 & 1521 & $1.23 \times 10^{-5}$ & 1 & TGTGAGTTATCTCACT & +627 & TTHC002 & $S$ \\
\hline 79,627 & 79,642 & $1.30 \times 10^{-5}$ & 1 & TGTGGTCCAGGCTACC & -78 & TTHB089 & $1 / 3$ \\
\hline 79,627 & 79,642 & $1.30 \times 10^{-5}$ & 1 & GGTAGCCTGGACCACA & -162 & ТTHB088 & S \\
\hline 615,132 & 615,147 & $1.46 \times 10^{-5}$ & 1 & GGTAGCCAGGGATACA & +909 & TTHA0647 & $4 / 4$ \\
\hline $1,715,061$ & $1,715,076$ & $1.65 \times 10^{-5}$ & 1 & TGTAGGCCAGGCCACG & -33 & TTHA1833 & $1 / 2$ \\
\hline 609,145 & 609,160 & $1.83 \times 10^{-5}$ & 1 & CGTGTCCCTGAACACA & +790 & TTHA0641 & $2 / 4$ \\
\hline 614,143 & 614,158 & $2.12 \times 10^{-5}$ & 1 & TGTGCCTTTGGCCACA & +326 & TTHA0645 & $1 / 3$ \\
\hline $1,794,923$ & $1,794,938$ & $2.33 \times 10^{-5}$ & 1 & GGTATGCTCAAGTACA & +13 & TTHA1912 & $1 / 2$ \\
\hline $1,794,923$ & $1,794,938$ & $2.33 \times 10^{-5}$ & 1 & TGTACTTGAGCATACC & -19 & TTHA1911 & $1 / 4$ \\
\hline 1272 & 1287 & $2.61 \times 10^{-5}$ & 1 & TGTAGCCCAGGCCAAA & +239 & ТТНВ003 & S \\
\hline 1272 & 1287 & $2.61 \times 10^{-5}$ & 1 & TTTGGCCTGGGCTACA & +536 & ТTHB004 & $4 / 4$ \\
\hline 199,120 & 199,135 & $2.90 \times 10^{-5}$ & 1 & TGTGGCGTATAACAAA & -17 & TTHA0202 & S \\
\hline 199,120 & 199,135 & $2.90 \times 10^{-5}$ & 1 & TTTGTTATACGCCACA & -103 & ТTHА0201 & S \\
\hline 357,035 & 357,050 & $3.43 \times 10^{-5}$ & 1 & AGTGATGTAAACTAAA & -26 & TTHA0374 & S \\
\hline 314,103 & 314,118 & $3.67 \times 10^{-5}$ & 1 & TGTGTTGCAGGACCCA & +58 & ТTHА0326 & $2 / 11$ \\
\hline $1,540,358$ & $1,540,373$ & $3.95 \times 10^{-5}$ & 1 & TGTAGCTTCCCATACC & -67 & TTHA1627 & S \\
\hline $1,540,358$ & $1,540,373$ & $3.95 \times 10^{-5}$ & 1 & GGTATGGGAAGCTACA & +13 & TTHA1626 & S \\
\hline
\end{tabular}

( $p$-Value) The probability of a random sequence of the same length matching that position of the sequence with an as good or better score. ( $Q$-value) False discovery rate if the occurrence is accepted as significant. (Loc) Location of the TTHB099-binding site relative to the start site of transcription. (Gene) Proximal gene downstream of TTHB099 consensus sequence. (Op) Gene position within the postulated operon. (S) No operon, single transcriptional unit. 
>ITHA0080, complement(81208 ... 81623)

GCGCTCCGCGTCGGAGAGGACCTCGTAGGCGTCGTGCCAGCCCTCCGGGCCCACCACCT GTCCAGGCGGTCCAACACGGTGCGGGCGTCCACGTAGGGGACCACCAAGGCCCGCTTCT GTCCCGGGAGAGGGCTTCCACGCGCCACTGCACCTCCCCCGGGGGAAAGGGTTCGGCCAG ITTCCGCCAGACTTCGTCCATGTGITTTAGTTTACTTTAGGTTGCTCTCACCCCAAAGCC TTGGGGGAAGGCGAAGATGGGGGCATGAAGCGGTGGCTGGCGTTCCTTCCCTTCCTGGCC CTGGCCTGGGCTTTGGAGCTCAGGGTCACCGCCTCCTTGGTGGTGGACCTCTTCCCCCAG GCGGTGGTGGTGGAGCGGGTTACCGAGCCCAGGGGATCGTGGTGGTTTACCAGGC

$>$ ITHA0506, complement(472003 .. 472418)

GCCCGCCTGGGCCAGGGCCCTGAGGAGGCGGTAGAGGGTGCTCTTGGCGAGGCCCACCCG TTCGGCCAAGGGGCCCAAGGGGCTTTCCCCCGCCTGGGCCAGGGCCTCGAGGACCCGAA CCCCCTCTCCAGGGTCTTCACCGCCTGGGGCGGCTTCTCCCGAAGGACGCGCCATGCCGCT TAGGGTAACGGGGGCGGCCCTGTTTTTCAAGATACAAAAAATCTTTTTGCTTCTTGACA TCCCGCCCCGCCTCCCGTAAGCTCGGACCACCATGAAGGGCGTGGAGATCCGGAAAGACC ACCCCCTCCTGAAGGAGGTCCTGACGGAGGAGGCCCTGAGGTTCGTGGTGGCGCTGCACC GGGAGTTCAACCCGGTGCGCAAGGCCCTCCTGGAGCGGCGTCAAGCGCTTTGGGAG

>ITHA0132, complement (129522 .. 130201)

TGGGGATGGCCGTGGCCCCAAGGGCCTCCACCTCCGAGGCCACCCCCGTGGCGAGCTCCA CGTCGGGGTCCACGGCGATGACCGTGGCCCCGTTGCGCCCGTACCCGTGGGCGATGGCCC GCCCGAACCCCCGGCCCGCGCCCGTGACCATGACGATCTTCCCCTCGAGGCCCAGAAGG CCCGTGACATCACGGCCCATTGTAAGGGAGAATAAAGCCATGGCGCGCATCCGGGTGGTC CAAGGGGACATCACCGAGTTCCAAGGGGACGCCATCGTCAACGCCGCCAACAACTACCTG AAGCTCGGGGCCGGGGTGGCGGGGGGATCCTGAGGAAGGGCGGCCCCTCCATCCAGGAG GAGTGCGACCGCATCGGCAAGATCCGGGTGGGGGAGGCGGCGGTCACGGGGGCGGC

>ITHB088, complement(79427 ... 79842)

TTCACCAGGACGTCCACCGCCGGGGCGTCGGGGAGAGGTGGGCCACCCGCACCATGGCG CCTTGGCCCAGGGCCAGGCCGGCCAGGGCCGCCAGAACCAGAACAAAAAGGCCTCGTTTC ATCTTTTCACCTCCACGGGAAAAGCCTAGAGGGAGGCCTGCCCGTCAAAATGGGCGCAGG ATCTTTTCACCTCCACGGGAAAAGCCTAGAGGGAGGCCTGCCCGTCAAAATGGGCGCAGC TCGGGGGACCTTCGGCCCTAGCATCCTCCCAAAGGAGGTAAGGGCATGGACCGCAGGCGT TTTCTCACCGGTGCGGGGCTTTTTTTGGCGGCGGGAGGCCTTCCCTTGGGCCGGGCCCAG GGGCGCGCGCCCAAGGGGGTGAACGGGGGCGGCTTTTACCGCTTCCGGGTAGGGG

>TTHA1833, (1714861 .. 1715276)

AACCATCGTTCCCCTGAGGCAGGCCCTGGGCTTTAGGATCCTCGGGGCCTACTGGCTTTC CGAGCGGGAGTTCCTCTGGTTCGTGGCCCACGAGGACTTTGAGGAGGCGGAGAGAGCTTA CTACGCCCACCCCGAAAGGCAGAAGGTGGACCCCAGGGCGTACCTGGAGGCGGTGGAAAC CCGTTTCGTGGAACGCCTTCTGTAGGCCAGGCCACGCCCCTGGCCCCGCCTTGGGGTAG CTCGGAGGGATGGAGCTTTTCCTCCTCGTCCTCCGCAACCTCCTGGCCCGGCCCGTCCGG AGCCTCCTCACCCTGCTCGGGGTCCTGGTGGCCACGGCGAGCATGGTCCTCTTCCTCTC TTCGGGGAGGGCCTTAGGCGGGCCCTCTTCCAGGAGCTCTCCCGGGTGGGCCCCGC

>TTHA1912，(1794726 .. 1795150)

GATGA AGCTCCCGGCGATGGAGTCCACCACCATCACCTGGTCCACCTCCACCACCACGAGCTCC CCGCCCGCACGGGCCTTCCCACCTTGTGGGAGAGGATCTTTTCCGCTAGCGTCTGTCCC CGGACACCTCCTATACTGAGGGTATGCTCAAGTACACCGCCCTCCTCTACCCGGACCCG AGACCCCAGGGGTCTGGATCGCCGAGTTTCCCGCGGTGCCCCAAGCCCACTCCTTCGGC AAAGCCCCGAGGAAGCCTTTGCGCGGGCCAAGAAGCCCTGGAGCTCGTCCTG TGAAAACCGAGGGGCGCCCCCTTCCCCAGGACGTACAAGCGGTAGAGGTAGGTGTC

$>$ ITHAO202, (198920 .. 199335)

GGGCGTAGCTGGGAAGCCCCGGGCTAACGTCCACCTCCACGGTGACGGGAACCGCGTCCA GGCCGAAGAGGGCGTAGCTTCGCACCTGGGCCAGCATGGAGAGAGTTTATCACAGCGCT TTAGTTCCACCCAAGGTGGGCGTTTCGTGAGCAGAGGCGAAAACTGCCTTATCATGGGG CAGATGGCGCGCGAGCTCCATGTGGCGTATAACAAAATGCGCCGCGCCCTGGAGGAGCGC TTGGGCCTCCTCCGCCGCCTCGGGGAATGGACCTCCGCTTGATCCAAGTGGCCAACGAG GAGTGGCTCTACATGCTCCAGGAGGACACCCGCAACTCCCTGGCCATAGAGGGCTACTTC ACCACGGAGCGGGAGCTACGGGAGGTGCTTAGGGGACGCAAGGGGGCGGCGGAGGT

$>$ TTHA1626, complement(1540158 .. 1540573)

CCGTGCCCGACTGGGGCAGGCCCTCCACCCCCAAGGTGGCGTCCAAGGGGGTGGGGGCGA GGGCGAGGAGGAAGGGGAAGAGGTCCTCCAAGGCTCCTCCCAGGCCCAGGCCGTCCACGA AGAGGAAGAGCACCTTTCCATCCTAAGGGGGGACATTTGCCACAGGGGGATAGAGGTACC CTGAGCTTAGGAGGTGATGGGGTATGGGAAGCTACAACCCGCTGGTCTTCGTTCTAGGCC TGGTCACGGCGGCCGGGGTCTCGGGGGTGGCCTACTTGCTCGCCGTGGCCCGGGGTGGGG ACGAGAAGGCCCTGGGGCGGCTTTATGGCCCCCTCTTCTTCACCCTGGGGTCTTCTCCC TGGGGGCGGTGGCCCAGCTCTACTGGACCAACTGGGCGGGCCGTCCGGTGCCCCAG
>TTHA0081, (81208 .. 81623)

GCCTGGTAAACCACCACGATCCCCTGGGGCTCGGTAACCCGCTCCACCACCACCGCCTGC GGGAAGAGGTCCACCACCAAGGAGGCGGTGACCCTGAGCTCCAAAGCCCAGGCCAGGGCC AGGAAGGGAAGGAACGCCAGCCACCGCTTCATGCCCCCATCTTCGCCTTCCCCCAAGGC TTGGGGTGAGAGCAACCTAAAGTAAACTAAAACACATGGACGAAGTCTGGCGGAAACTGG CCGAACCCTTTCCCCCGGGGGAGGTGCAGTGGCGCGTGGAAGCCCTCTCCCGGGACAAGA AGCGGGCCTTGGTGGTCCCCTACGTGGACGCCCGCACCGTGTTGGACCGCCTGGACAAGG TGGTGGGCCCGGAGGGCTGGCACGACGCCTACGAGGTCCTCTCCGACGCGGAGCGC

\section{>TTHA0507, (472003 …4472418)}

CTCCCAAAGCGCTTGACGCCGCTCCAGGAGGGCCTTGCGCACCGGGTTGAACTCCCGGTG CAGCGCCACCACGAACCTCAGGGCCTCCTCCGTCAGGACCTCCTTCAGGAGGGGGTGGT TTTCCGGATCTCCACGCCCTTCATGGTGGTCCGAGCTTACGGGAGGCGGGCGGGATTG CAAGAAGCAAAAAGATTTTTTGTATCTTGAAAAACAGGGCCGCCCCCGTTACCCTAAGCG GCATGGCGCGTCCTCGGGAGAAGCCGCCCCAGGCGGTGAAGACCCTGGAGAGGGGGCTTC GGGTCCTCGAGGCCCTGGCCCAGGCGGGGGAAAGCCCCTTGGGCCCCTTGGCCGAACGG TGGGCCTCGCCAAGAGCACCCTCTACCGCCTCCTCAGGGCCCTGGCCCAGGCGGGC

>TTHA0133, (129831 .. 130938)

CCCGCCCCCGTGACCGCCGCCTCCCCCACCCGGATCTTGCCGATGCGGTCGCACTCCTCC TGGATGGAGGGGCCGCCCTTCCTCAGGATCGCCCCCGCCACCCCGGCCCCGAGCTTCAGO TAGTTGTTGGCGGCGTTGACGATGGCGTCCCCTTGGAACTCGGTGAT GTCCCCTTGGACC ACCCGGATGCGCGCCATGGCTTTATTCTCCCTTACAATGGGCCGTGATGTCACGGGACCT TCTGGGCCTCGAGGGGAAGATCGTCATGGTCACGGGCGCGGGCCGGGGGTTCGGGCGGGC CATCGCCCACGGGTACGGGCGCAACGGGGCCACGGTCATCGCCGTGGACCCCGACGTGGA GCTCGCCACGGGGGTGGCCTCGGAGGTGGAGGCCCTTGGGGCCACGGCCATCCCCA

>TTHB089, (79427 ... 79842)

CCCCCTACCCGGAAGCGGTAAAAGCCGCCCCCGTTCACCCCCTTGGGCGCGCGCCCCTGG GCCCGGCCCAGGGAAGGCCTCCCGCCGCCAAAAAAGCCCCGACCGGGAGACGC CTGCGGTCCATGCCCTTACCTCCTITGGGAGGATGCTAGGGCCGAAGGTCCCCCGAGAAT CTGCGGTCCATGCCCTTACCTCCTTTGGGAGGATGCTAGGGCCGAAGGTCCCCCGAGAAT GTGCTCCCCCTCACCCTGGGTGTGGTCCAGGCTACCTTGGCGGGAGGTTTATGTGGCCT CGAGGCCTTTTTGTTCTGGTTCTGGCGGCCCTGGCCGGCCTGGCCCTGGGCCAAGGCGCC ATGGTGCGGGTGGCCCACCTCTCCCCCGACGCCCCGGCGGTGGACGTCCTGGTGAA

\section{>TTHA1911, complement(1794726 .. 1795150)}

CACACCTACCTCTACCGCTTGTACGTCCTGGGGAAGGGGGCGCCCCTCGGTTTTCAGATA GGCCAGGACGAGCTCCAGGGCTTCTTTGGCCCGCGCCAAGGCTTCCTCGGGGCTTTGGCC GAAGGAGTGGGCTTGGGGCACCGCGGGAAACTCGGCGATCCAGACCCCTGGGGTCTCCG GTCCGGGTAGAGGAGGGCGGTGTACTTGAGCATACCCTCAGTATAGGAGGTGTCCGTGG ACAGACGCTAGCGGAAAAGATCCTCTCCCACAAGGTGGGAAGGCCCGTGCGGGCGGGGGA GCTCGTGGTGGTGGAGGTGGACCAGGTGATGGTGGTGGACTCCATCGCCGGGAGCTTCTT CAAGCGCCTGGAGTACCTGGAGGCCACCCCCCGCTACCCGGAAAGGGTCTCCATCG

>ITHA0201, complement(198920 .. 199335)

ACCTCCGCCGCCCCCTTGCGTCCCCTAAGCACCTCCCGTAGCTCCCGCTCCGTGGTGAAG TAGCCCTCTATGGCCAGGGAGTTGCGGGTGTCCTCCTGGAGCATGTAGAGCCACTCCTC TTGGCCACT TGGATCAAGCGGAGGTCCATTCCCCCGAGGCGGCGGAGGAGGCCCAAGCG TCCTCCAGGGCGCGGCGCATTTTGTTATACGCCACATGGAGCTCGCGCGCCATCTGGCCC CATGATAAGGCAGTTTTCGCCTCTGCTCACGAAACGCCCACCTTGGGTGGAACTAACAGC GCTGTGATAAACTCTCTCCATGCTGGCCCAGGTGCGAAGCTACGCCCTCTTCGGCCTGGA CGCGGTTCCCGTCACCGTGGAGGTGGACGTTAGCCCGGGGCTTCCCAGCTACGCCC

>TTHA0374, (356835 .. 357250)

CAACAACGTGGATCCCGAGCGGGACGCCCGGGTGATGCCGGGGGTGGAGGGGCCGGTTT GGTCCTGGACGGCACGAGGAAGCTCCCCGAGGAGGGCTTCCCCAGGGTCTGGCCCGAGA GATCCGGATGGACCCCAAGGTGAAGGCCTTGGTGGAGGCCCGGTGGGCGGAGTACGGCC GGGCTGGACAACGGTGGGTGAGTGATGTAAACTAAAAGAGGTTTAGTGCGAAGGTGATAT TTATGGGCTTACACGTCCTCGGCGTGAACGCATCGGCTAGGACGGACGGTTTTACGGCGG AGCTTTTGACGAGGTTTTGGAGGCGGCAGGCGCAAGGGGGCGACCACCGAGCGCCTG ATTTGGTGCGGCACCCCTTTCCCCTCTGCGCCGGCAACTACTCCGTGGACCCCGCT

\section{> $\underline{\text { THA1627 }}$, (1540158 .. 1540573)}

CTGGGGCACCGGACGGCCCGCCCAGTTGGTCCAGTAGAGCTGGGCCACCGCCCCCAGGGA GAAGACCCCCAGGGTGAAGAAGAGGGGGCCATAAAGCCGCCCCAGGGCCTTCTCGTCCCC ACCCCGGGCCACGGCGAGCAAGTAGGCCACCCCCGAGACCCCGGCCGCCGTGACCAGGCC TAGAACGAAGACCAGCGGGTTGTAGCTTCCCATACCCCATCACCTCCTAAGCTCAGGGTA CCTCTATCCCCCTGTGGCAAATGTCCCCCCTTAGGATGGAAAGGTGCTCTTCCTCTTCGT CCTCTATCCCCCTGTGGCAAATGTCCCCCCTTAGGATGGAAAGGTGCTCTTCCTCTTCG GGACGGCCTGGGCCTGGGAGGAGCCTTGGAGGACCTCTTCCCCTTCCTCCTCGCCC
CCCCACCCCCTTGGACGCCACCTTGGGGGTGGAGGGCTTGCCCCAGTCGGGCACGG

Figure 6. Promoter predictions of sequences potentially regulated by TTHB099 within the T. thermophilus HB8 genome. Shown are $\pm 200 \mathrm{bp}$ sequences from the TTHB099 binding site identified through FIMO (see Table 2). Blue nucleotides represent the longest open reading frames with a downstream orientation relative to the TTHB099 binding site; Green nucleotides indicate open reading frames with the opposite orientation; Black nucleotides imply intergenic regions. Potential promoter elements ( -35 and -10 boxes, +1 start site of transcription) are indicated with cyan highlighting; TTHB099-binding sites are indicated with yellow highlighting; Overlapping TTHB099-binding and core promoter elements are indicated by green highlighting. 


\subsection{Validation of Potential TTHB099-Regulated Genes}

Apart from analyzing the locations of the binding sequences concerning the TSS, as well as their positions regarding promoters, we investigated the affinity of TTHB099 protein for the selected sequences. To better understand how TTHB099 regulates genes identified through FIMO, all 16 sequences underwent binding kinetics analysis using BLI. As some TTHB099 binding sites are shared by two bidirectional promoters, only nine unique sequences were synthesized into biotinylated double-stranded oligonucleotides. Binding reactions containing four different concentrations of TTHB099 (450, 150, 50, and $17 \mathrm{nM}$ ) were tested against each binding site probe (Table 3$)$. The strongest binding was observed for TTHA1833 and TTHB088/89, with $\mathrm{K}_{\mathrm{D}}$ values below $10 \mathrm{nM}$. The genes with binding affinities between 10-100 nM were TTHA1911/12, TTHA0506/07, and TTHA0080/81 in increasing order. TTHA1626/27, TTHA0132/33, and TTHA0201/02 displayed the weakest binding, with $\mathrm{K}_{\mathrm{Ds}}>100 \mathrm{nM}$, while binding to TTHA0374 could not be detected under our experimental conditions. Interestingly, these binding parameters do not always follow the sequence order defined by FIMO, suggesting that there could be other factors at play that are not considered by this in vitro analysis.

Table 3. Binding kinetics parameters of TTHB099 to potential gene promoter elements.

\begin{tabular}{cccccc}
\hline Gene & Sequence & $\boldsymbol{k}_{\text {on }}\left(\mathbf{M}^{-\mathbf{1}} \mathbf{s}^{-\mathbf{1}} \mathbf{)}\right.$ & $\boldsymbol{k}_{\mathbf{\text { off }}} \mathbf{( \mathbf { s } ^ { - \mathbf { 1 } } )}$ & $\mathbf{K}_{\mathbf{D}} \mathbf{( \mathbf { M } )}$ & $\mathbf{R}^{\mathbf{2}}$ \\
\hline TTHA0080/81 & TGTGTTTTAGTTTACT & 122,852 & $1.145 \times 10^{-2}$ & $9.322 \times 10^{-8}$ & 0.9817 \\
TTHA0506/07 & TGTTTTTCAAGATACA & 164,971 & $1.280 \times 10^{-2}$ & $7.762 \times 10^{-8}$ & 0.9718 \\
TTHA0132/33 & TGTAAGGGAGAATAAA & 96,736 & $2.140 \times 10^{-2}$ & $2.212 \times 10^{-7}$ & 0.9687 \\
TTHB088/89 & GGTAGCCTGGACCACA & 214,153 & $7.163 \times 10^{-4}$ & $3.345 \times 10^{-9}$ & 0.9805 \\
TTHA1833 & TGTAGGCCAGGCCACG & 332,611 & $1.013 \times 10^{-3}$ & $3.046 \times 10^{-9}$ & 0.9757 \\
TTHA1911/12 & TGTACTTGAGCATACC & 136,294 & $8.938 \times 10^{-3}$ & $6.558 \times 10^{-8}$ & 0.9806 \\
TTHA0201/02 & TTTGTTATACGCCACA & 57,231 & $4.464 \times 10^{-2}$ & $7.801 \times 10^{-7}$ & 0.9596 \\
TTHA0374 & AGTGATGTAAACTAAA & - & - & - & - \\
TTHA1626/27 & GGTATGGGAAGCTACA & 126,605 & $1.291 \times 10^{-2}$ & $1.020 \times 10^{-7}$ & 0.9759 \\
\hline
\end{tabular}

(TTHA0080/81) A common TTHB099-binding site shared by two bidirectional promoters. (-) No apparent binding.

Further validation of TTHB099 involvement in the transcriptional regulation of these genes was sought through the analysis of prior DNA microarray studies, publicly available through the National Center for Biotechnology Information Gene Expression Omnibus (NCBI GEO) [24]. A GEO2R comparison (SuperSeries GSE21875, Supplementary Table S2) of expression profile data from sets of TTHB099-deficient and wild type strains was used to determine if the absence of TTHB099 produced any substantial changes in the expression of the FIMO-identified genes. Of these genes, only TTHA1626 displayed a substantially increased expression with a $\operatorname{logFC}$ of 2.62. The remainder of the 15 genes had only small, non-significant changes, as shown in Table 4. Likewise, individual genes within their respective operons did not seem to have any significant changes.

As an additional approach to better understand potential gene regulation by TTHB099, we investigated the postulated biological functions of these genes. Many were reported only as encoding hypothetical proteins, which is fairly common in T. thermophilus. Several encoded proteins that may be involved in sugar metabolism (malate synthase, 3-isopropylmalate dehydratase), energy metabolism (3-isopropylmalate dehydratase large subunit, homoaconitate hydratase small subunit), or transport. Most interesting, two genes (TTHA0134 and TTHA0507) are believed to encode transcriptional regulators. If so, their expression could complicate the identification of directly TTHB099-regulated genes by GEO2R. 
Table 4. Expression profile data of the FIMO identified operons in a TTHB099-deficient strain of T. thermophilus HB8.

\begin{tabular}{|c|c|c|c|c|}
\hline Operon & Gene & Role & $\operatorname{LogFC}$ & Adj. $p$-Value \\
\hline$S$ & TTHA0080 & hypothetical protein & 0.851 & 0.0268 \\
\hline 1 & TTHA0081 & hypothetical protein & -0.202 & 0.421 \\
\hline 2 & TTHA0082 & phosphoesterase & -0.176 & 0.463 \\
\hline 3 & TTHA0083 & dimethyladenosine transferase & -0.219 & 0.336 \\
\hline S & TTHA0506 & malate synthase & -0.454 & 0.0983 \\
\hline S & TTHA0507 & $\begin{array}{l}\text { IclR family transcriptional regulator, } \\
\text { acetate operon repressor }\end{array}$ & 0.276 & 0.619 \\
\hline$S$ & TTHA0132 & hypothetical protein & 0.872 & 0.0295 \\
\hline 1 & ТTHА0133 & $\begin{array}{l}\text { Short-chain dehydrogenase/ } \\
\text { reductase family oxidoreductase }\end{array}$ & -0.211 & 0.674 \\
\hline 2 & TTHA0134 & NrdR family transcriptional regulator & -0.328 & 0.350 \\
\hline$S$ & TTHB088 & Zn-dependent hydrolase & -0.386 & 0.653 \\
\hline 1 & ТTHB089 & hypothetical protein & -0.779 & 0.0451 \\
\hline 2 & ТТНВ090 & hypothetical protein & -0.0653 & 0.955 \\
\hline 3 & ТТHB091 & hypothetical protein & -0.217 & 0.674 \\
\hline 1 & TTHA1833 & $\mathrm{ABC}$ transporter permease & -0.294 & 0.287 \\
\hline 2 & TTHA1834 & $\mathrm{ABC}$ transporter ATP-binding protein & -0.195 & 0.567 \\
\hline 1 & TTHA1911 & 3-isopropylmalate dehydratase large subunit & -0.817 & 0.0246 \\
\hline 2 & TTHA1910 & homoaconitate hydratase small subunit & -1.14 & 0.0265 \\
\hline 3 & TTHA1909 & hypothetical protein & -0.0793 & 0.790 \\
\hline 4 & TTHA1908 & hypothetical protein & -0.0327 & 0.905 \\
\hline 1 & TTHA1912 & hypothetical protein & 0.353 & 0.154 \\
\hline 2 & TTHA1913 & hypothetical protein & 0.723 & 0.0284 \\
\hline$S$ & TTHA0201 & $\mathrm{Mg}^{2+}$ chelatase family protein & 0.141 & 0.698 \\
\hline$S$ & TTHA0202 & hypothetical protein & 0.454 & 0.0644 \\
\hline$S$ & TTHA0374 & hypothetical protein & 0.687 & 0.0421 \\
\hline$S$ & TTHA1626 & hypothetical protein & 2.62 & $2.10 \times 10^{-3}$ \\
\hline S & TTHA1627 & hypothetical protein & -1.20 & 0.0960 \\
\hline
\end{tabular}

(Operon) Numbers indicate positions of the genes within the operon. (S) Single transcriptional unit. (Role) The biological function identified using the KEGG database [19]. (LogFC) Log2-fold change between data obtained from TTHB099-deficient (accessions GSM530118/20/22) and wild-type (accessions GSM532194/5/6) T. thermophilus HB8 strains, SuperSeries GSE21875. (Adj. $p$-value) The $p$-value obtained following multiple testing corrections using the default Benjamini and Hochberg false discovery rate method [25].

Another analysis of the GEO2R data was focused on investigating the genes that were affected most by the absence of TTHB099 (Table 5). These genes could be grouped into operons, suggesting that their expression was not affected by multiple-unrelated TFs, but rather a fundamental regulatory mechanism involving TTHB099. The upregulated genes, 75\% (50/67), were involved in the electron transport chain (ETC) of oxidative phosphorylation, carbohydrate metabolism, bacteria motility, and osmotic stress defense. The downregulated operons, $25 \%$ (17/67 genes), were related to ribosomal proteins, ion ABC transporters, and ATPases. MEME analysis of the $-300 /+100$ bp sequences upstream of each operon did not find our TTHB099 consensus sequence or reveal any additional binding motifs. Taken together, this suggests a complicated mechanism for the regulation of these genes that may not involve TTHB099 directly regulating their transcription. 
Table 5. GEO2R analysis of the most affected genes in the absence of TTHB099.

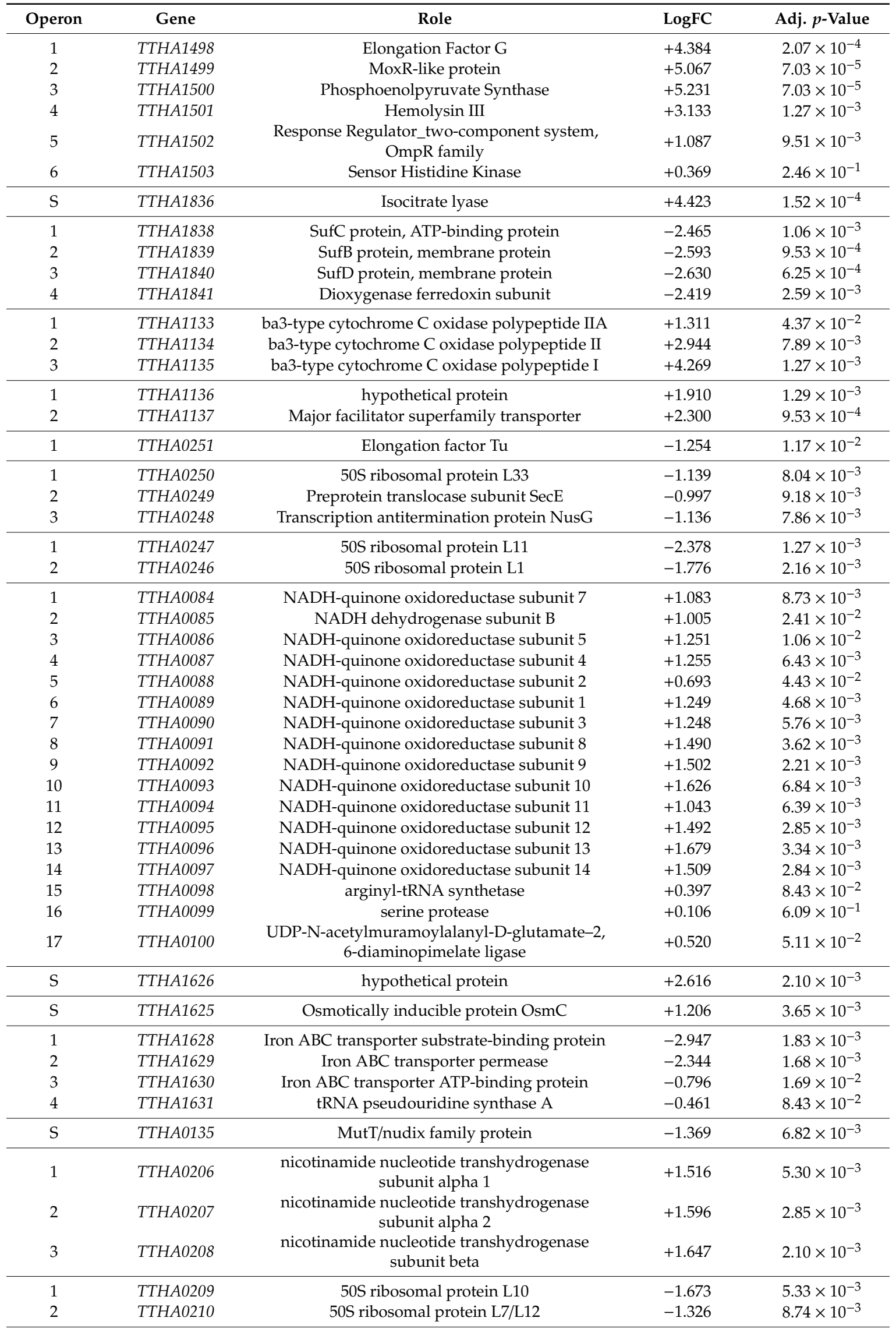


Table 5. Cont.

\begin{tabular}{ccccc}
\hline Operon & Gene & Role & LogFC & Adj. $\boldsymbol{p}$-Value \\
\hline 1 & TTHB117 & putative type IV pilin & +1.125 & $4.09 \times 10^{-2}$ \\
2 & TTHB118 & secretion system protein & +1.450 & $3.74 \times 10^{-3}$ \\
3 & TTHB119 & hypothetical protein & +1.429 & $5.85 \times 10^{-3}$ \\
4 & TTHB120 & +2.250 & $1.27 \times 10^{-3}$ \\
\hline 1 & TTHA1652 & maltose ABC transporter substrate-binding protein & +1.787 & $1.72 \times 10^{-3}$ \\
2 & TTHA1651 & maltose ABC transporter permease & +2.154 & $1.17 \times 10^{-3}$ \\
3 & TTHA1650 & maltose ABC transporter permease & +2.108 & $1.29 \times 10^{-3}$ \\
\hline 1 & TTHB186 & putative transcriptional regulator & +3.377 & $2.59 \times 10^{-3}$ \\
2 & TTHB187 & hypothetical protein & +2.036 & $7.58 \times 10^{-3}$ \\
\hline 1 & TTHB188 & hypothetical protein & +1.215 & $9.19 \times 10^{-3}$ \\
2 & TTHB189 & CRISPR-associated Cse2 family protein & +1.514 & $4.80 \times 10^{-3}$ \\
3 & TTHB190 & hypothetical protein & +1.671 & $6.62 \times 10^{-3}$ \\
4 & TTHB191 & hypothetical protein & +1.480 & $4.34 \times 10^{-3}$ \\
5 & TTHB192 & hypothetical protein & +1.669 & $4.68 \times 10^{-3}$ \\
6 & TTHB193 & hypothetical protein & +1.446 & $6.84 \times 10^{-3}$ \\
7 & TTHB194 & hypothetical protein & +1.549 & $1.71 \times 10^{-2}$ \\
\hline
\end{tabular}

(Operon) Numbers indicate positions of the genes within the operon. (S) Single transcriptional unit. (Role) The biological function identified using the KEGG database (LogFC) Log2-fold change between data obtained from TTHB099-deficient and wild-type T. thermophilus HB8 strains. (Adj. p-value) The $p$-value obtained following multiple testing corrections using the default Benjamini and Hochberg false discovery rate method [25].

\section{Discussion}

In this study, an in vitro iterative selection method, REPSA, was used to annotate the TTHB099 transcription regulator in T. thermophilus HB8. This, coupled with next generation sequencing and MEME motif elicitation, allowed for the identification of the TTHB099-DNA binding motif, a $16 \mathrm{bp}$ long palindromic sequence, $5^{\prime}-\mathrm{TGT}(\mathrm{A} / \mathrm{g}) \mathrm{n}(\mathrm{t} / \mathrm{c}) \mathrm{c}(\mathrm{t} / \mathrm{c})(\mathrm{a} / \mathrm{g}) \mathrm{g}(\mathrm{a} / \mathrm{g}) \mathrm{n}(\mathrm{T} / \mathrm{c}) \mathrm{ACA}-3^{\prime}$, with a consensus half-site $5^{\prime}-\mathrm{T}_{1} \mathrm{G}_{2} \mathrm{~T}_{3}(\mathrm{~A} / \mathrm{G})_{4} \mathrm{~N}_{5}(\mathrm{~T} / \mathrm{C})_{6} \mathrm{C}_{7}(\mathrm{~T} / \mathrm{C})_{8}-3^{\prime}$. Binding kinetics between TTHB099 and its consensus sequence, as well as single point mutations within its half-site, were investigated using BLI. TTHB099 protein bound the 16-mer consensus sequence with a high affinity $\left(K_{\mathrm{D}}=2.21 \mathrm{nM}\right)$ and the point-mutated sequences in the range of 4.86 of $33.6 \mathrm{nM}$ with mutations at the second and third positions having the greatest effect. The different binding affinities for each mutated sequence mirrored the MEME results represented by the TTHB099 sequence logo. Our report is the first time a consensus sequence has been identified for TTHB099.

Interestingly, our sequence has a strong resemblance to the E. coli $\mathrm{CRP}\left(\mathrm{CRP}_{\mathrm{Ec}}\right)$ consensus sequence, 5'-AAATGTGATCTAGATCACATTT-3' [26]. In both cases, the trimers "TGT" and "ACA" are highly conserved and are considered most significant for TF binding. The specifics of this resemblance could be correlated to the homology between the two proteins previously reported by Agari et al. [12]. However, E. coli and T. thermophilus HB8 are not only phylogenetically distant, but they also live in entirely different environments, mesophilic and extremophilic, respectively [27]. Hence, the biological roles of TTHB099 need not necessarily be the same as those of $\mathrm{CRP}_{\mathrm{Ec}}$. This is most evident in the observation that TTHB099 does not require the second messenger $3^{\prime}, 5^{\prime}$ cAMP to bind DNA, which is required by $\mathrm{CRP}_{\mathrm{Ec}}$.

Having found and validated a consensus TTHB099-binding sequence, mapping it onto the genome of T. thermophilus HB8 would help identify potential TTHB099-regulated genes. Using FIMO, the MEME derived position weight matrix version of our consensus sequence recognized 78 sequences. The top 25 sequences with the best $p$-values were selected for further validation. It is important to note that the $p$-values derived were not as small as found in our previous studies, due to the ten poorly conserved positions in the middle of the TTHB099 consensus sequence palindrome, which affected the dynamic programming algorithm of FIMO. Our analysis of the TTHB099 binding site location relative to the TSS of the proximal downstream genes showed that almost half of the identified sites were located inside open reading frames, which is not typical for traditional transcription factors. Notably, no potential 
TTHB099 binding site was found near its own gene. This could imply that the TTHB099 TF by itself has no direct regulatory role over its operon litR (TTHB100, TTHB099, TTHB098) or the divergent crtB operon (TTHB101, TTHB102) that shares a common intergenic region. Autoregulation is a common feature for many prokaryotic TFs, including members of the CRP family, but may not be a characteristic for TTHB099 unless in an auxiliary fashion [28].

The promoter analysis revealed that nine TTHB099-binding sites overlapped with potential core promoter elements, a TF-promoter interaction characteristic of Class II transcription activators, as well as transcription inhibition via steric hindrance. Additionally, three sequences are located upstream of the -35 box, fitting the Class I activator model, while two are downstream of the -10 box, a model used by both transcription activators and repressors. These variations in the binding method suggest that TTHB099 could be either an activator or a suppressor. Indeed, the dual regulatory role is common in global regulators such as $\mathrm{CRP}_{\mathrm{Ec}}$ [29]. Moreover, eight pairs of the TTHB099-binding sequences were found in the intergenic region of divergent genes, another characteristic of dual-regulators [30].

Biophysical studies performed with BLI were used to further our understanding of TTHB099 interaction with the identified sites. The equilibrium dissociation constants were below the micromolar range, showing that TTHB099 had some appreciable affinity for the tested sites. However, variations as high as 200 -fold were observed. These $K_{D}$ changes did not follow any particular trends, such as the $p$-value order established through FIMO. Neither did the sites with the highest affinity have similarities in terms of promoter location or presumed manner of transcription regulation. For example, the TTHB099 binding sequence with the highest affinity $(3.05 \mathrm{nM})$ was located in the intergenic region and overlapped with the -35 box upstream of TTHA1833. The TTHB099 binding sequences with the next lowest $\mathrm{K}_{\mathrm{D}}$ were also situated in the intergenic regions, but they were located upstream and downstream of the TTHB088/89 promoters, respectively. Such biophysical results emphasize the importance of experimental validation of theoretically determined sites.

Our BLI binding studies are limited to the simple interactions of purified protein with synthesized DNAs in the absence of any environmental or biological factors. Knowing that the transcription regulation apparatus can be complex, we decided to complement our in vitro study with data from in vivo expression profiles. Using publicly available expression profile data from the matched wild type and TTHB099-deficient T. thermophilus HB8 strains, operons of the 16 potentially regulated genes were investigated. We found that the mRNAs of these genes were not significantly affected by the deficiency of TTHB099. These results suggest that TTHB099 does not have, on its own, any appreciable regulatory roles over these genes in exponentially propagating wild type organisms.

Nonetheless, TTHB099 deficiency does appreciably affect the expression of several genes in exponentially propagating T. thermophilus HB8. We identified 19 operons, 12 of which were overexpressed (positively affected) in the deficient strains. The upregulated set of genes were involved in the electron transport chain (ETC) of oxidative phosphorylation, sugar metabolism, type IV pilin related proteins, and one osmotically inducible protein, consistent with TTHB099 being a transcriptional repressor. Conversely, there were seven under-expressed operons or a total of 17 genes in the TTHB099-deficient strains, suggesting that TTHB099 may act as an activator for these genes. The downregulated genes encoded for ribosomal proteins, iron ABC transporters, and ATPases. Notably, the biological roles of the most affected operons in the TTHB099-deficient strain were involved in metabolic pathways that have been reported to be regulated by the archetype $\mathrm{CRP}_{\mathrm{Ec}}$ [31]. For example, ribosome related genes were downregulated in the absence of TTHB099, similar to what Pal et al. reported for their evolutionary expressed $\mathrm{CRP}_{\mathrm{Ec}}$-deficient strains [32]. Likewise, iron transport genes were downregulated in the absence of TTHB099, similar to what was observed in the absence of CRP $\mathrm{Ec}_{\mathrm{Ec}}$ as Zhang et al. reported [33]. Such results indicate that TTHB099 does have some biological functions similar to those of the $\mathrm{CRP}_{\mathrm{Ec}}$. However, these regulatory roles do not seem to be affected by changes in cAMP concentration. Moreover, a MEME search for a consensus sequence between the 19 most-affected operons identified via the GEO data failed to bring up any significant motifs. Thus, the hypothesis for a simple regulatory mechanism is once more unsatisfied. 
TT_P0055 from T. thermophilus HB27, an ortholog of TTHB099 with only one amino acid substitution (E77D), has been reported to be a positive regulator of $c r t B$ operon, which in turn is involved in light-dependent carotenoid biosynthesis [33]. However, the functional effects of TT_P0055 on carotenoid production lack details on the mechanism of regulation and could indicate that TT_P0055 has indirect control over crtB activation. The homology between the HB27 and HB8 strains, particularly on this regulatory complex (TT_P0055 and TTHB099 proteins, their intergenic regions, and their $\operatorname{crtB}$ operons), would suggest similar biological functions for the two TFs. When analyzing the GEO expression data in the absence of TTHB099, there is no detectable change in $c r t B$ genes. These results could be attributed to the absence of light in the experimental conditions required to deplete the litR transcriptional repressor of TT_P0055, the latter positively regulating carotenoid production [34].

Because TTHB099 does not seem to have any observable binding to the $\mathrm{crtB}$ promoter, the study published by Ebright et al. centered on TTHB099 binding upstream of TTHB101 is based on a prediction not firmly established [35]. Hence, Ebright's claim that TTHB099 is a model class II transcription activator may need to be reconsidered under the light of our new findings.

Looking for a connection between the genes found via the REPSA-identified consensus sequence and the genes affected by TTHB099 deficiency, as determined by GEO2R, we found that five of the affected operons (30 genes) had an upstream binding sequence identified by FIMO. Interestingly, these binding sites were located at about 0.9 to $4 \mathrm{kbp}$ upstream of the most affected operons. Such behavior could be explained by TTHB099 acting as an enhancer or silencer. These elements do exist in the prokaryotic world but not in large numbers. To date, the identified prokaryotic enhancers regulate only a few promoters used by $\sigma^{54}$-directed RNA polymerases [36]. Knowing that T. thermophilus HB8 does not have a $\sigma^{54}$ homolog, it becomes even more challenging to suggest that TTHB099 can function as an enhancer/silencer. Future studies could be designed to analyze potential interactions of TTHB099 with other TFs, supporting the hypothesis of a complex regulatory mechanism involving distal enhancer/silencer elements. As for TTHB099 being an activator or a suppressor, all our data point towards a dual regulatory role.

\section{Materials and Methods}

\subsection{Preparation of Oligonucleotides}

Single-stranded oligonucleotides used in this study (Supplementary Table S3) were obtained from Integrated DNA Technologies (Coralville, IA). ST2R24 library DNA used for the initial REPSA round was PCR amplified with primers ST2L and IRD7_ST2R for seven cycles to ensure maximal double-stranded DNA content with fully annealed randomized cassette regions. Subsequent REPSA round DNAs were PCR amplified for 6, 9, and 12 cycles to identify those products with optimal cassette integrity. Libraries for massively parallel semiconductor sequencing were prepared by a two-step fusion PCR process, using primers A_BC01_ST2R and trP1_ST2L as the initial set and A_uni and trP1_uni as the second set, as previously described [8]. Other duplex DNAs were prepared by conventional PCR amplification following the Taq DNA polymerase manufacturer's instructions. EMSA probes were amplified with primers ST2L and IRD7_ST2R, while nucleic acids used in BLI assays were amplified with primers ST2L and Bio_ST2R. The concentrations for the modified oligonucleotides were measured with Qubit 3 Fluorometer following our protocol [37].

\subsection{TTHB099 Protein Expression and Purification}

TTHB099 protein was expressed following IPTG induction of E. coli BL21(DE3) bacteria transformed with plasmid PC014099-42 (obtained from RIKEN Bioresource Research Center) and purified from soluble bacterial extracts by heat-treatment as described in our previous study [11]. SDS-PAGE analysis of fractions from purification steps is shown in Supplementary Figure S2A and is consistent with a near quantitative recovery of TTHB099 protein. Analysis by quantitative densitometry with Coomassie 
Brilliant Blue staining indicated that the purified TTHB099 preparation had a final concentration of $50.6 \mu \mathrm{M}$ (Supplementary Figure S2B).

\subsection{TTHB099-Consensus Sequence Determination}

REPSA selections with $50.6 \mathrm{nM}$ TTHB099 were performed essentially as previously described [8], with the exception that 3.2 U FokI were used in Rounds 1-4 and $8 \mathrm{U}$ BpmI were used in Rounds 5-7. Furthermore, the Round 1 reactions were seeded with $4.515 \mathrm{ng}(100 \mathrm{fmol})$ ST2R24 DNA pool. The PCR amplification reactions were adjusted to use $560 \mathrm{nM}$ of primers and $25 \mathrm{U}$ NEB Taq polymerase. Finally, the annealing and elongation temperatures were adjusted to $58^{\circ} \mathrm{C}$ and $68{ }^{\circ} \mathrm{C}$, respectively.

The amplicon library preparation, Ion PGM individual sequencing particle (ISP) preparation, Ion PGM semiconductor sequencing, and Ion Torrent sever sequence processing were all performed as previously described [8]. Resulting raw sequences in fastq format (Supplementary Data S1) were further processed by our Sequencing1.java program [8] and DuplicatesFinder v 1.1 (http: //proline.bic.nus.edu.sg/ asif/tools/DuplicateFinder.zip) to yield data (Supplementary Data S2) suitable for consensus sequence determination by web version 5.0.5 of Multiple Em for Motif Elicitation (MEME) (http://meme-suite.org/tools/meme) [15]. Position-weight matrices for the top three motifs were determined and displayed as sequence logos, from which a consensus sequence was derived.

\subsection{Protein-DNA Binding Assays}

Electrophoretic mobility shift assays (EMSA) with both libraries and defined DNA were performed as previously described [8], with a detailed protocol being available [38]. Note that EMSA experiments performed with REPSA selected DNAs contain multiple DNA species, including high concentrations of DNA primers, and should not be used to determine apparent binding affinities. Biolayer interferometry was performed as previously described [11], with the exception that only four concentrations of TTHB099 $(17,50,150,450 \mathrm{nM})$ were used for each DNA probe investigated. Such was sufficient to yield global values for $k_{\text {on }}$ and $k_{\text {off }}$ rate constants as well as $\mathrm{K}_{\mathrm{D}}$ equilibrium binding constants with $\mathrm{R}^{2}$ goodness-of-fit determinations of greater than 0.95 in all cases. A single BLI experiment was performed with $2.25 \mathrm{nM}$ consensus (wt) probe, $200 \mathrm{nM}$ TTHB099, and $100 \mathrm{nM} \mathrm{3} 3^{\prime}, 5^{\prime}$-cAMP, to test the effects of cAMP on TTHB099-DNA binding. Its $\mathrm{R}^{2}$ value was 0.92 .

\subsection{Bioinformatic Determination of Candidate Regulated Genes}

The 16-bp position weight matrix obtained from a MEME analysis of our processed sequencing data was used as the input for FIMO analysis (http://meme-suite.org/tools/fimo) [18], to identify best matches within the T. thermophilus genome. Stringency was limited to include matches having $p$-values $\leq 3.95 \times 10^{-5}$. Sequences $\pm 200 \mathrm{bp}$ from the TTHB099 binding site were analyzed by Softberry BPROM (http://www.softberry.com) [23] to identify potential bacterial core promoter elements. Operons were identified using the Database of PrOkaryotic OpeRons (DOOR ${ }^{2}$ ) (http://161.117.81. 224/DOOR3/annotate.php) and BioCyc (http://biocyc.org) [21,22]. Putative biological functions of TTHB099-regulated genes were obtained using T. thermophilus HB8 data from KEGG (https://www. genome.jp/kegg-bin/show_organism?org=T00220) [19]. Publicly available microarray data for gene expression profiles in wild-type and TTHB099-deficient T. thermophilus HB8 were obtained from the NCBI GEO website (https://www.ncbi.nlm.nih.gov/geo/) SuperSeries GSE21875 [24]. In particular, samples GSM532194, 5, and 6, obtained from wild-type T. thermophilus HB8 grown in a rich medium for $360 \mathrm{~min}$ and samples GSM530118, 20, and 22, obtained from TTHB099-deficient T. thermophilus HB8 strains propagated under identical conditions. These data sets were analyzed using their NCBI GEO2R program with default settings to determine changes in gene expression (LogFC values) and their statistical significance ( $p$-values). 
Supplementary Materials: The following are available online at http://www.mdpi.com/1422-0067/21/21/7929/s1, Table S1: EMSA quantification data, Table S2: GEO2R analysis of expression profiles from TTHB099-deficient and wild type T. thermophilus HB8 strains, Table S3: Oligonucleotides, Figure S1: Quantitative densitometry analysis of TTHB099 binding to its palindromic consensus sequence, Figure S2: Expression, purification, and quantification of TTHB099 protein, Data S1: REPSA Round 7 fastq sequences, and Data S2: REPSA Round 7 Sequencing1-processed sequences can be found at http://www.mdpi.com/1422-0067/21/21/7929/s1.

Author Contributions: Conceptualization, M.W.V.D.; investigation, K.M. and M.W.V.D.; formal analysis, K.M., M.W.V.D.; data curation, K.M. and M.W.V.D.; writing-original draft, K.M. and M.W.V.D.; writing-eview and editing, K.M. and M.W.V.D.; supervision, M.W.V.D.; project administration, M.W.V.D.; funding acquisition, M.W.V.D. All authors have read and agreed to the published version of the manuscript.

Funding: This work was supported by the US National Science Foundation (Grant MCB 1714778), and the Kennesaw State University Foundation (Foundation Fellow in Biochemistry).

Conflicts of Interest: The authors declare no conflict of interest. In addition, the funders had no role in the design of the study; in the collection, analyses, or interpretation of data; in the writing of the manuscript, or in the decision to publish the results.

\section{Abbreviations}

$\begin{array}{ll}\text { BLI } & \text { Biolayer Interferometry } \\ \text { cAMP } & 3^{\prime}, 5^{\prime} \text {-cyclic Adenosine Monophosphate } \\ \text { CRP } & \text { Cyclic AMP Receptor Protein } \\ \text { DOOR } & \text { Database of PrOkaryotic OpeRons } \\ \text { EMSA } & \text { Electrophoretic Mobility Shift Assay } \\ \text { ETC } & \text { Electron Transport Chain } \\ \text { FIMO } & \text { Find Individual Motif Occurrences } \\ \text { GEO } & \text { Gene Expression Omnibus } \\ \text { IISRE } & \text { Type IIS Restriction Endonuclease } \\ \text { IRD7 } & \text { IRDye }{ }^{\circledR} \text { 700 } \\ \text { ISP } & \text { Individual Sequencing Particle } \\ \text { KEGG } & \text { Kyoto Encyclopedia of Genes and Genomes } \\ \text { MEME } & \text { Multiple Em for Motif Elicitation } \\ \text { NCBI } & \text { National Center for Biotechnology Information } \\ \text { PGM } & \text { Personal Genome Machine } \\ \text { REPA } & \text { Restriction Endonuclease Protection Assay } \\ \text { REPSA } & \text { Restriction Endonuclease Protection, Selection, and Amplification } \\ \text { TetR } & \text { Tetracycline Repressor Protein } \\ \text { TF } & \text { Transcription Factor } \\ \text { TFBS } & \text { Transcription Factor Binding Site } \\ \text { TSS } & \text { Transcription Start Site } \\ & \end{array}$

\section{References}

1. Collado-Vides, J.; Magasanik, B.; Gralla, J.D. Control site location and transcriptional regulation in Escherichia coli. Microbiol. Mol. Biol. Rev. 1991, 55, 371-394. [CrossRef]

2. Mejía-Almonte, C.; Busby, S.J.; Wade, J.T.; van Helden, J.; Arkin, A.P.; Stormo, G.D.; Eilbeck, K.; Palsson, B.O.; Galagan, J.E.; Collado-Vides, J. Redefining fundamental concepts of transcription initiation in bacteria. Nat. Rev. Genet. 2020, 21, 1-16. [CrossRef] [PubMed]

3. $\mathrm{Xu}, \mathrm{H} . ;$ Hoover, T.R. Transcriptional regulation at a distance in bacteria. Curr. Opin. Microbiol. 2001, 4, 138-144. [CrossRef]

4. Browning, D.F.; Busby, S.J. The regulation of bacterial transcription initiation. Nat. Rev. Microbiol. 2004, 2, 57-65. [CrossRef] [PubMed]

5. Martínez-Antonio, A.; Collado-Vides, J. Identifying global regulators in transcriptional regulatory networks in bacteria. Curr. Opin. Microbiol. 2003, 6, 482-489. [CrossRef] [PubMed]

6. Price, M.N.; Dehal, P.S.; Arkin, A.P. Orthologous transcription factors in bacteria have different functions and regulate different genes. PLoS Comput. Biol. 2007, 3, e175. [CrossRef] [PubMed] 
7. Santos-Zavaleta, A.; Salgado, H.; Gama-Castro, S.; Sánchez-Pérez, M.; Gómez-Romero, L.; Ledezma-Tejeida, D.; García-Sotelo, J.S.; Alquicira-Hernández, K.; Muñiz-Rascado, L.J.; Peña-Loredo, P. RegulonDB v 10.5: Tackling challenges to unify classic and high throughput knowledge of gene regulation in E. coli K-12. Nucleic Acids Res. 2019, 47, D212-D220. [CrossRef]

8. Van Dyke, M.W.; Beyer, M.D.; Clay, E.; Hiam, K.J.; McMurry, J.L.; Xie, Y. Identification of preferred DNA-binding sites for the Thermus thermophilus transcriptional regulator SbtR by the combinatorial approach REPSA. PLoS ONE 2016, 11, e0159408. [CrossRef]

9. Lee, M.; Um, H.; Van Dyke, M.W. Identification and characterization of preferred DNA-binding sites for the Thermus thermophilus transcriptional regulator FadR. PLoS ONE 2017, 12, e0184796. [CrossRef]

10. Cox, J.S.; Moncja, K.; Mckinnes, M.; Van Dyke, M.W. Identification and Characterization of Preferred DNA-Binding Sites for the Thermus thermophilus HB8 Transcriptional Regulator TTHA0973. Int. J. Mol. Sci. 2019, 20, 3336. [CrossRef]

11. Shell Cox, J.; Van Dyke, M.W. General and Genomic DNA-Binding Specificity for the Thermus thermophilus HB8 Transcription Factor TTHB023. Biomolecules 2020, 10, 94.

12. Agari, Y.; Kuramitsu, S.; Shinkai, A. X-ray crystal structure of TTHB099, a CRP/FNR superfamily transcriptional regulator from Thermus thermophilus HB8, reveals a DNA-binding protein with no required allosteric effector molecule. Proteins 2012, 80, 1490-1494. [CrossRef] [PubMed]

13. Hardenbol, P.; Van Dyke, M.W. Sequence specificity of triplex DNA formation: Analysis by a combinatorial approach, restriction endonuclease protection selection and amplification. Proc. Natl. Acad. Sci. USA 1996, 93, 2811-2816. [CrossRef] [PubMed]

14. Van Dyke, M.; Gracien, I. Restriction endonuclease protection assays using infrared-fluorescent probes. Protocols.io 2020, bi5ikg4e. [CrossRef]

15. Bailey, T.L.; Elkan, C. Fitting a mixture model by expectation maximization to discover motifs in biopolymers. Proc. Int. Conf. Intell. Syst. Mol. Biol. 1994, 2, 28-36.

16. Concepcion, J.; Witte, K.; Wartchow, C.; Choo, S.; Yao, D.; Persson, H.; Wei, J.; Li, P.; Heidecker, B.; Ma, W.; et al. Label-free detection of biomolecular interactions using BioLayer interferometry for kinetic characterization. Comb. Chem. High Throughput Screen. 2009, 12, 791-800. [CrossRef]

17. Popovych, N.; Tzeng, S.R.; Tonelli, M.; Ebright, R.H.; Kalodimos, C.G. Structural basis for cAMP-mediated allosteric control of the catabolite activator protein. Proc. Natl. Acad. Sci. USA 2009, 106, 6927-6932. [CrossRef]

18. Grant, C.E.; Bailey, T.L.; Noble, W.S. FIMO: Scanning for occurrences of a given motif. Bioinformatics 2011, 27, 1017-1018. [CrossRef]

19. Kanehisa, M.; Sato, Y.; Furumichi, M.; Morishima, K.; Tanabe, M. New approach for understanding genome variations in KEGG. Nucleic Acids Res. 2019, 47, D590-D595. [CrossRef]

20. Brown, G.R.; Hem, V.; Katz, K.S.; Ovetsky, M.; Wallin, C.; Ermolaeva, O.; Tolstoy, I.; Tatusova, T.; Pruitt, K.D.; Maglott, D.R. Gene: A gene-centered information resource at NCBI. Nucleic Acids Res. 2015, 43, D36-D42. [CrossRef]

21. Mao, F.; Dam, P.; Chou, J.; Olman, V.; Xu, Y. DOOR: A database for prokaryotic operons. Nucleic Acids Res. 2009, 37, D459-D463. [CrossRef] [PubMed]

22. Karp, P.D.; Billington, R.; Caspi, R.; Fulcher, C.A.; Latendresse, M.; Kothari, A.; Keseler, I.M.; Krummenacker, M.; Midford, P.E.; Ong, Q. The BioCyc collection of microbial genomes and metabolic pathways. Brief. Bioinform. 2019, 20, 1085-1093. [CrossRef]

23. Solovyev, V.; Salamov, A. Automatic annotation of microbial genomes and metagenomic sequences. In Metagenomics and Its Applications in Agriculture, Biomedicine and Environmental Studies; Li, R.W., Ed.; Nova Science Publishers: Hauppauge, NY, USA, 2011; pp. 61-78.

24. Barrett, T.; Wilhite, S.E.; Ledoux, P.; Evangelista, C.; Kim, I.F.; Tomashevsky, M.; Marshall, K.A.; Phillippy, K.H.; Sherman, P.M.; Holko, M.; et al. NCBI GEO: Archive for functional genomics data sets-Update. Nucleic Acids Res. 2013, 41, D991-D995. [CrossRef]

25. Benjamini, Y.; Hochberg, Y. Controlling the false discovery rate: A practical and powerful approach to multiple testing. J. R. Stat. Soc. Ser. B 1995, 57, 289-300. [CrossRef]

26. Leuze, M.R.; Karpinets, T.V.; Syed, M.H.; Beliaev, A.S.; Uberbacher, E.C. Binding motifs in bacterial gene promoters modulate transcriptional effects of global regulators CRP and ArcA. Gene Regul. Syst. Biol. 2012, 6, 93-107. [CrossRef] 
27. Hartmann, R.K.; Wolters, J.; Kröger, B.; Schultze, S.; Specht, T.; Erdmann, V.A. Does Thermus represent another deep eubacterial branching? Syst. Appl. Microbiol. 1989, 11, 243-249. [CrossRef]

28. Aiba, H. Autoregulation of the Escherichia coli crp gene: CRP is a transcriptional repressor for its own gene. Cell 1983, 32, 141-149. [CrossRef]

29. Galan-Vasquez, E.; Sanchez-Osorio, I.; Martinez-Antonio, A. Transcription factors exhibit differential conservation in bacteria with reduced genomes. PLoS ONE 2016, 11, e0146901. [CrossRef]

30. Balleza, E.; Lopez-Bojorquez, L.N.; Martínez-Antonio, A.; Resendis-Antonio, O.; Lozada-Chávez, I.; Balderas-Martínez, Y.I.; Encarnación, S.; Collado-Vides, J. Regulation by transcription factors in bacteria: Beyond description. FEMS Microbiol. Rev. 2008, 33, 133-151. [CrossRef]

31. Shimada, T.; Fujita, N.; Yamamoto, K.; Ishihama, A. Novel roles of cAMP receptor protein (CRP) in regulation of transport and metabolism of carbon sources. PLoS ONE 2011, 6, e20081. [CrossRef]

32. Pal, A.; Iyer, M.S.; Srinivasan, S.; Seshasayee, A.S.; Venkatesh, K.V. Elucidating the regulatory role of CRP in coordinating protein biosynthesis machinery with metabolism that defines growth optimality in Escherichia coli. bioRxiv 2020. [CrossRef]

33. Zhang, Z.; Gosset, G.; Barabote, R.; Gonzalez, C.S.; Cuevas, W.A.; Saier, M.H. Functional interactions between the carbon and iron utilization regulators, Crp and Fur, in Escherichia coli. J. Bacteriol. 2005, 187, 980-990. [CrossRef] [PubMed]

34. Takano, H.; Kondo, M.; Usui, N.; Usui, T.; Ohzeki, H.; Yamazaki, R.; Washioka, M.; Nakamura, A.; Hoshino, T.; Hakamata, W. Involvement of CarA/LitR and CRP/FNR family transcriptional regulators in light-induced carotenoid production in Thermus thermophilus. J. Bacteriol. 2011, 193, 2451-2459. [CrossRef]

35. Feng, Y.; Zhang, Y.; Ebright, R.H. Structural basis of transcription activation. Science 2016, 352, 1330-1333. [CrossRef]

36. Bondarenko, V.; Liu, Y.; Ninfa, A.; Studitsky, V.M. Action of prokaryotic enhancer over a distance does not require continued presence of promoter-bound $\sigma 54$ subunit. Nucleic Acids Res. 2002, 30, 636-642. [CrossRef]

37. Van Dyke, M. Direct Double-Stranded DNA Quantitation from PCR Reactions V.2. Protocols.io 2017, k5pcy5n. [CrossRef]

38. Van Dyke, M.; Cox, J. Electrophoretic mobility shift assays using infrared-fluorescent DNA probes. Protocols.io 2018, mbdc2i6. [CrossRef]

Publisher's Note: MDPI stays neutral with regard to jurisdictional claims in published maps and institutional affiliations.

(C) 2020 by the authors. Licensee MDPI, Basel, Switzerland. This article is an open access article distributed under the terms and conditions of the Creative Commons Attribution (CC BY) license (http://creativecommons.org/licenses/by/4.0/). 